\title{
N-of-1 Trials as a Decision Support Tool in Clinical Practice: A Protocol for a Systematic Literature Review and Narrative Synthesis
}

\author{
Joyce Samuel $^{1, *}$, Travis Holder ${ }^{2}$ and Donald Molony ${ }^{1}$ \\ 1 McGovern Medical School, The University of Texas Health Science Center at Houston, 6431 Fannin St, \\ Houston, TX 77030, USA; donald.a.molony@uth.tmc.edu \\ 2 Houston Academy of Medicine, The Texas Medical Center Library, 1133 John Freeman Blvd, Houston, \\ TX 77030, USA; travis.holder@library.tmc.edu \\ * Correspondence: joyce.samuel@uth.tmc.edu; Tel.: +1-713-500-6708
}

Received: 29 August 2019; Accepted: 2 November 2019; Published: 6 November 2019

\begin{abstract}
The n-of-1 trial can utilized in clinical practice as a decision support tool, which may improve patient outcomes by providing both the patient and the clinician with objective evidence to inform personalized treatment decisions. As its use broadens, it will be important to study whether the added time and effort of an n-of- 1 trial results in measurable improvements in important patient outcomes compared to usual clinical practice. Parallel-group randomized clinical trials testing the n-of-1 approach versus usual care have been undertaken in a number of medical settings. A systematic review will be performed according to PRISMA guidelines, using MEDLINE, Embase, Cochrane, CINAHL, PsycINFO, Scopus, and Web of Science to search for randomized clinical trials in humans, without date or language restriction. Reports from the gray literature and ongoing studies in trial registries will be included. Articles will be screened by two independent reviewers with a third reviewer consulted to adjudicate disagreement. The quality of included studies will be assessed using the Cochrane Collaboration's tool for assessing risk of bias. A narrative synthesis will explore the differing methodological approaches of the included studies. The protocol will be registered in the PROSPERO registry, and the results of the review will be published in a peer-reviewed journal. To our knowledge, this systematic review will be the first to comprehensively assess the existing research on randomized trials testing the n-of-1 trial approach in clinical practice.
\end{abstract}

Keywords: n-of-1 trial; single case experimental design; personalized trial

\section{Introduction}

N-of-1 trials, or personalized trials, represent an innovative scientific strategy that can be viewed simultaneously as both research and clinical practice. One of the fundamental principles of evidence-based medicine is that clinical practice should begin with an understanding of the best available unbiased evidence. Answers to treatment questions can be garnered from parallel-group randomized clinical trials (RCTs), in which large groups of individuals are exposed to either the intervention in question or a control condition, and the average response of the groups is carefully measured and compared. However most clinicians are all too familiar with the limitations of this approach; the most important being (1) the dearth of RCTs undertaken in many of the conditions and populations that they are responsible for treating, and (2) the question of generalizability-that the individual's response to a treatment may vary from the observed average treatment effect in an RCT. Given the remaining uncertainty inherent in clinical practice, n-of- 1 trials have the potential to play an important role in patient care. In an n-of-1 trial, an individual patient is exposed to one or more interventions and a control condition in random order, and the individual's response is 
carefully measured to estimate the relative treatment effects for the individual. N-of- 1 trials can be used in clinical practice as a decision support tool by clinicians who must decide between two or more therapeutic alternatives for a chronic condition [1]. The ultimate goal of the n-of- 1 trial as a clinical tool is to produce evidence to inform treatment plans that have been tailored to the individual based on his or her unique responses to the therapies under consideration.

A number of case reports and case series describing the use of n-of- 1 trials across a diverse spectrum of disease states and interventions have been published since their introduction to the medical landscape by Guyatt and colleagues in 1986 [2-5].

In usual clinical practice, prescribing decisions are often unsystematic and informed primarily by clinician preferences, anecdotal experience, and trial-and-error. Conversely, when used as a decision support tool, n-of- 1 trials represent a treatment approach that reduces the guesswork inherent in usual practice. A variety of techniques can be employed to minimize bias, including randomized treatment order, objective outcomes assessment, and repeated treatment periods. Widespread use of n-of-1 trials in the clinical setting has been hindered by multiple factors, including a lack of awareness of the approach and skepticism about whether the n-of- 1 trial results in measurable improvements in important patient outcomes compared to usual clinical practice, especially in light of the additional time and effort that may be required.

A systematic review of prospective randomized clinical trials is the gold standard in establishing whether any given intervention is superior to the alternative [6]. In this case, the intervention in question is the use of $n$-of- 1 trials as a decision tool, and the alternative is usual clinical practice. The medical literature will be searched for RCTs testing groups of individuals whose treatment decisions were informed by either an n-of- 1 trial or usual clinical practice.

The primary objective is to conduct a systematic review of the literature to determine whether $\mathrm{n}$-of- 1 trials have been shown in randomized trials to improve clinical outcomes compared to usual care. We will also critically appraise the methodological approaches of included studies to assess whether scientific rigor can be improved for future studies.

\section{Materials and Methods}

This protocol was developed based on the Preferred Reporting Items for Systematic Reviews and Meta-Analysis for Protocols (PRISMA-P) 2015 statement, and will be registered in the PROSPERO international prospective registry [7]. Any important protocol amendments will be reported when publishing the results. The systematic review will be performed and reported according to the Preferred Reporting Items for Systematic Reviews and Meta-Analysis (PRISMA) guidelines [8].

\subsection{Eligibility Criteria}

We will search the medical literature for prospective, parallel-group, randomized clinical trials (RCTs) that compare a group of human subjects who were randomized to the n-of- 1 trial strategy with another group who were randomized to another treatment approach and followed over time to assess a difference in any health outcome. We will restrict our search to medical n-of- 1 trials. No restriction will be placed on language or date.

The terminology used to designate n-of- 1 of trials may be inconsistent across the body of literature on this topic. We will include only those RCTs testing n-of-1 trials that meet the following criteria: (1) randomized treatment periods within blocks or pairs, (2) crossover of interventions, (3) single patients as the unit of analysis for the n-of- 1 trial.

We will exclude case reports or case series of n-of- 1 trials, n-of- 1 observational designs, studies describing behavioral interventions, and expert reviews and commentaries.

\subsection{Information Sources}

The following databases will be electronically searched from inception: MEDLINE (PubMed), Embase, Cochrane Library, CINAHL, PsycINFO, Scopus, and Web of Science. We will search the gray literature using the Open Grey database and by consultation with experts. Unpublished or ongoing 
studies will be identified by searching ClinicalTrials.gov and the WHO international clinical trials registry platform. The reference lists of all included studies will be hand-searched.

\subsection{Search Strategy}

A comprehensive search strategy is under development and will include (but not be limited to) "n-of-1", "personalized trial", "single case experimental design", "multi-period crossover design", and "single subject trial." A second search will be conducted translating identified keywords, index terms, and necessary commands into each database.

\subsection{Data Management and Study Selection}

References will be managed in the screening and selection process using Rayyan, a web-based application [9]. After duplicates are removed, two investigators will screen all retrieved records by titles and subsequently by abstract, using the eligibility criteria described above. Disagreements over eligibility at the title and abstract level will be discussed first and persistent disagreements will be arbitrated by a third reviewer. For all articles with abstracts determined to be eligible, the full text will be retrieved and evaluated by two reviewers independently, with disagreements handled in the same manner as the title and abstract screening step. A flow diagram demonstrating the search and selection process will be developed according to PRISMA guidelines.

\subsection{Data Collection}

A standardized data extraction form will be developed and 2 investigators will independently extract the following data (see Table 1) regarding the overall RCT and the design of the individual n-of- 1 trials separately.

Table 1. Data items to be extracted.

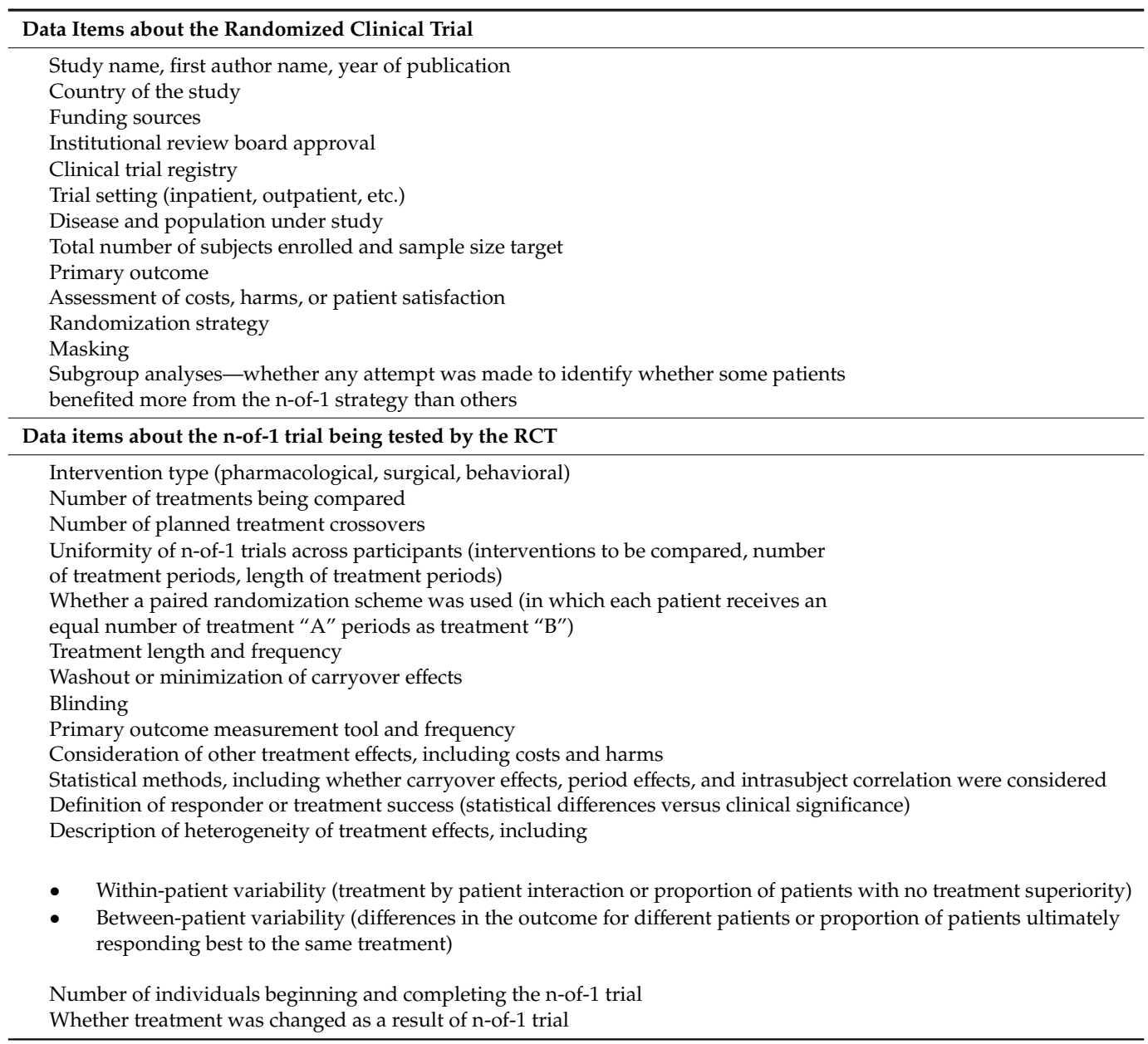




\subsection{Outcomes and Prioritization}

All included studies will be summarized in a table of study characteristics, and we anticipate including the following main outcomes: primary outcome and whether comparison between groups was a summary of individual responses to treatment versus aggregated group response; acceptability of n-of- 1 trial, including rates of drop-out and patient satisfaction; and cost analysis.

\subsection{Risk of Bias in Individual Studies}

Assessment of the completeness of the reporting will be measured using the Consolidated Standards of Reporting Trials (CONSORT) extension for n-of-1 trials (CENT) checklist [10]. For each $\mathrm{RCT}$, the risk of bias will be assessed using the domain-based Cochrane Collaboration tool to evaluate risk of bias in randomized trials [11].

\subsection{Data Synthesis}

Quantitative meta-analysis will not be appropriate or possible due to anticipated differences in population, diagnoses, and interventions across included studies. We will use a narrative approach to provide summary descriptions of n-of-1 trial design, RCT study design, study quality, risk of bias, and outcomes reported.

\subsection{Meta-Biases and Confidence in Cumulative Evidence}

Risk of publication bias will be discussed, including why n-of-1 trials are particularly vulnerable due to inconsistent protocol registration. The Grading of Recommendations Assessment, Development and Evaluation (GRADE) criteria will be used to summarize the strength of the evidence to support the use of n-of-1 trials [12].

\section{Discussion}

To our knowledge, this review represents the first attempt to systematically review the body of evidence pertaining to the rigorous testing of n-of- 1 trials against usual practice using RCTs. This systematic review will be submitted for publication in a peer-reviewed journal. The results will be used to identify whether more trials are needed, and may provide insight into how future RCTs may be designed with improved rigor to advance the science of n-of- 1 trials.

Author Contributions: J.S. conceptualized and designed the protocol and wrote the manuscript, T.H. designed the search strategy and reviewed and revised the manuscript, D.M. assisted in study design and critically reviewed and revised the manuscript.

Funding: This work was supported by the Clinical and Translational Sciences Award (1UL1TR003167-01) from the NIH National Center for Advancing Translational Sciences.

Conflicts of Interest: The authors declare no conflict of interest. The funding body had no role in the design of the study; in the collection, analyses, or interpretation of data; in the writing of the manuscript, or in the decision to publish.

\section{References}

1. Nikles, C.J.; Clavarino, A.M.; Del Mar, C.B. Using n-of-1 trials as a clinical tool to improve prescribing. Br. J. Gen. Pr. 2005, 55, 175-180.

2. Guyatt, G.; Sackett, D.; Taylor, D.W.; Ghong, J.; Roberts, R.; Pugsley, S. Determining Optimal Therapy-Randomized Trials in Individual Patients. N. Engl. J. Med. 1986, 314, 889-892. [CrossRef] [PubMed]

3. Gabler, N.B.; Duan, N.; Vohra, S.; Kravitz, R.L. N-of-1 trials in the medical literature: A systematic review. Med. Care 2011, 49, 761-768. [CrossRef]

4. Punja, S.; Bukutu, C.; Shamseer, L.; Sampson, M.; Hartling, L.; Urichuk, L.; Vohra, S. N-of-1 trials are a tapestry of heterogeneity. J. Clin. Epidemiol. 2016, 76, 47-56. [CrossRef] [PubMed] 
5. Barlow, D.H.; Nock, M.K. Why Can't We Be More Idiographic in Our Research? Perspect. Psychol. Sci. 2009, 4, 19-21. [CrossRef]

6. OCEBM Levels of Evidence Working Group. The Oxford Levels of Evidence 2. Oxford Centre for Evidence-Based Medicine. Available online: https://www.cebm.net/index.aspx?o=5653 (accessed on 26 August 2019).

7. Shamseer, L.; Moher, D.; Clarke, M.; Ghersi, D.; Liberati, A.; Petticrew, M.; Shekelle, P.; Stewart, L.A. The PRISMA-P Group Preferred reporting items for systematic review and meta-analysis protocols (PRISMA-P) 2015: Elaboration and explanation. BMJ 2015, 349, g7647. [CrossRef]

8. Moher, D.; Liberati, A.; Tetzlaff, J.; Altman, D.G. Preferred Reporting Items for Systematic Reviews and Meta-Analyses: The PRISMA Statement. PLoS Med. 2009, 6, e1000097. [CrossRef] [PubMed]

9. Ouzzani, M.; Hammady, H.; Fedorowicz, Z.; Elmagarmid, A. Rayyan-a web and mobile app for systematic reviews. Syst. Rev. 2016, 5, 210. [CrossRef] [PubMed]

10. Vohra, S.; Shamseer, L.; Sampson, M.; Bukutu, C.; Schmid, C.H.; Tate, R.; Nikles, J.N.; Zucker, D.R.; Kravitz, R.; Guyatt, G.; et al. CONSORT extension for reporting N-of-1 trials (CENT) 2015 Statement. BMJ 2015, 350, h1738. [CrossRef] [PubMed]

11. Higgins, J.P.T.; Altman, D.G.; Gøtzsche, P.C.; Jüni, P.; Moher, D.; Oxman, A.D.; Savović, J.; Schulz, K.F.; Weeks, L.; Sterne, J.A.C. The Cochrane Collaboration's tool for assessing risk of bias in randomised trials. BMJ 2011, 343, d5928. [CrossRef] [PubMed]

12. Guyatt, G.H.; Oxman, A.D.; Vist, G.E.; Kunz, R.; Falck-Ytter, Y.; Alonso-Coello, P.; Schünemann, H.J. GRADE: An emerging consensus on rating quality of evidence and strength of recommendations. BMJ 2008, 336, 924-926. [CrossRef] [PubMed]

(C) 2019 by the authors. Licensee MDPI, Basel, Switzerland. This article is an open access article distributed under the terms and conditions of the Creative Commons Attribution (CC BY) license (http://creativecommons.org/licenses/by/4.0/). 